\title{
What next for the junior doctor contract?
}

Aoife Abbey is a specialist trainee in intensive care, not anaesthesia, as we put in this Feature (BMJ 2016;352:i991, doi:10.1136/bmj.i991). We apologise for this error.
Cite this as: BMJ 2016;352:11088

๑ BMJ Publishing Group Ltd 2016 